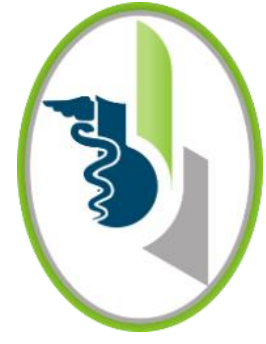

ACCESO $\odot$ ABIERTO

Para citaciones: Boneu, D., Barragán, R., Arroyo, D. (2021). Asociación entre valsartán e inhibidores de neprilisina en el manejo de la falla cardiaca. Revista Ciencias Biomédicas, 10(3), 198-206.

https://doi.org/10.32997/rcb-2021-3500

Recibido: 23 de marzo de 2021

Aprobado: 3 de junio de 2021

Autor de correspondencia:

Deiby Boneu Yepez

diboneu@hotmail.com

Editor: Inés Benedetti. Universidad de Cartagena-Colombia.

\section{Asociación entre valsartán e inhibidores de neprilisina en el manejo de la falla cardiaca}

\author{
Association between valsartan and neprilisin inhibitors in the \\ management of heart failure
}

Deiby Boneu Yepez ${ }^{1}$ (D), Robinson Barragán Montiel² $^{2}$ Dayana Arroyo Carrascal²

${ }^{1}$ Áreas Básicas. Facultad de Ciencias de la Salud. Corporación Universitaria Rafael Núñez. Cartagena, Colombia.

${ }^{2}$ Pregrado Medicina, Corporación Universitaria Rafael Núñez, Cartagena, Colombia.

\section{RESUMEN}

Introducción: la falla cardiaca es una de las principales causas de muerte de origen cardiovascular y constituye la enfermedad cardiovascular con más alta tasa de rehospitalización en las personas ancianas. Se ha demostrado, con ayuda de varios ensayos clínicos, que el novedoso tratamiento LCZ696 para la falla cardiaca, compuesto de valsartán e inhibidores de neprilisinas, tiene un resultado mucho más eficaz que el tratamiento con Enalapril solamente.

Objetivo: analizar cómo actúa la combinación de valsartán e inhibidores de neprilisina en el manejo de la falla cardiaca.

Métodos: se revisaron los resúmenes y se escogieron los artículos que mostraran la terapia innovadora para falla cardiaca, basados en el mecanismo de inhibición de la Neprilisina. Se incluyeron los estudios sin restricción de diseño, con buen nivel de evidencia científica.

Resultados: se recolectaron artículos de las bases de datos: Pubmed, Ebscohost, Ovid, Cochrane Library, Scielo en español y Science Direct. Se obtuvieron en total 199 artículos que tratan sobre inhibidores de Neprilisina, 162 de los cuales puntualizaban sobre inhibidores de Neprilisina en el tratamiento de la falla cardiaca. Se obtuvieron 111 artículos completos de los que se incluyeron 39 artículos con información concerniente a la falla cardiaca, su etiología, clasificaciones, tratamiento innovador, entre otros temas.

Conclusión: Sacubitril/Valsartán es un medicamento que ha demostrado su beneficio en el tratamiento de la falla cardiaca.

Palabras Clave: Insuficiencia Cardíaca; Valsartán; Neprilisina; Péptido Natriurético Atrial; Inhibidores de la Enzima Convertidora de Angiotensina.

\section{ABSTRACT}

Introduction: hearth failure is one of the main causes of cardiovascular death and is the cardiovascular disease with the highest rate of rehospitalization in the elderly. Several clinical trials has been shown with the novel LCZ696 treatment 
for heart failure, consisting of valsartan and neprilysin inhibitors, which has a much more effective outcome than treatment with Enalapril alone.

Objective: to analyze how the combination of valsartan and neprilysin inhibitors works in the management of heart failure.

Methods: the abstracts were reviewed and articles of any modality presenting innovative therapy for heart failure, based on the inhibition mechanism of Neprilysin were selected. Were included studies without design restriction, with a good level of scientific evidence.

Results: articles were collected from Pubmed, Ebscohost, Ovid, Cochrane Library, Scielo in Spanish and ScienceDirect. A total of 199 articles dealing with Neprilysin inhibitors were obtained, 162 articles pointed to Neprilysin inhibitors for heart failure treatment. A total of 111 articles were obtained, 39 articles were included, which included subjects related to heart failure, its etiology, classifications, innovative treatment to treat heart failure, among others topics.

Conclusions: Sacubitril/Valsartan is a drug that has demonstrated its benefit in the heart failure treatment.

Keywords: Heart failure, Valsartan; Neprilysin; Atrial Natriuretic Factor; Angiotensin-Converting Enzyme Inhibitors.

\section{INTRODUCCIÓN}

La insuficiencia cardiaca, también conocida como falla cardiaca, es un síndrome cardiovascular, caracterizado por cursar con un conjunto de signos y síntomas que se presentan como el mal funcionamiento del corazón. Podemos deducir que existe una falla cardiaca cuando el corazón es incapaz de bombear la cantidad suficiente de sangre que requieren los tejidos para su metabolismo. Esta puede presentarse en uno de los ventrículos o en ambos, y puede deberse por problemas de eyección ventricular o en el llenado auricular, al producirse un gasto cardiaco insuficiente, el organismo activa una serie de mecanismos compensadores cardiovasculares y neurohormonales que tienen como función tratar de restaurar el equilibrio homeostático del corazón en su función como bomba.(1)

Como mecanismo neurohormonal de compensación se habla de la hipótesis que refiere acerca de la activación del sistema renina angiotensina aldosterona (SRAA), el cual se inicia por el daño al miocardio causado por factores etiopatogénicos como la hipertensión arterial o la isquemia, que hacen que el miocardio no responda de manera fisiológica, alterando su funcionalidad como bomba de expulsión y reduciendo su volumen de eyección una vez disminuye este volumen se da la estimulación de los barorreceptores ubicados en el cayado de la aorta y las carótidas, activando así el sistema nervioso simpático, que a su vez, libera noradrenalina para aumentar el inotropismo y el cronotropismo cardiaco; a nivel renal se activa el sistema RAA, a esto se le suma la vasoconstricción periférica apoyado por vasopresina, endotelina $\mathrm{y}$ angiotensina II (2) que contribuyen a que el organismo compense la aparente disminución de la volemia; de esta manera el flujo sanguíneo periférico disminuye y el flujo cardiaco aumenta a modo de compensación (3). Mientras tanto a nivel renal, en la arteriola aferente disminuye la presión sanguínea, provocando un estímulo que permite la liberación de renina para producir aldosterona que contribuirá a la retención de agua y sodio para así, aumentar la volemia (4). El objetivo es analizar cómo actúa la combinación de valsartán e inhibidores de neprilisina en el manejo de la falla cardiaca. 


\section{MÉTODOS}

Se revisaron las bases de datos PUBMED, COCHRANE, OVID, SCIELO EN ESPAÑOL, HINARI y EBSCOHOST. La búsqueda se realizó desde julio 2020 hasta noviembre 2020. Se obtuvieron artículos desde el año de 1984 hasta el año 2020 en idiomas inglés y español. Se incluyeron todos los artículos obtenidos, sin distingo de modalidad, tipo de estudio o investigación. Los términos clave fueron: Insuficiencia Cardíaca, Valsartán, Neprilisina, Péptido Natriurético Atrial, Inhibidores de la Enzima Convertidora de Angiotensina presentes en el DeCS. Se revisaron los resúmenes, se escogieron los artículos de cualquier modalidad, sin restricción de diseño y un nivel de evidencia que presentaban la terapia innovadora para el tratamiento de la falla cardiaca con base a la acción farmacológica de inhibición de la Neprilisina.

\section{RESULTADOS}

Al revisar las diferentes bases de datos, se obtuvieron los siguientes artículos: Pubmed 35545, Ebscohost 3074, Ovid 6869, Cochrane Library 1306, Scielo en español 74 y Science Direct 3179 artículos. Se escogieron 199 artículos que tratan sobre inhibidores de Neprilisina. De estos, 162 artículos puntualizaban sobre inhibidores de Neprilisina en el tratamiento de la insuficiencia cardiaca. Se seleccionaron 111 artículos completos, actualizados; de estos, se estudiaron 39 artículos que encerraban la información necesaria para completar el cuerpo teórico de este trabajo, que contenían temas concernientes a la falla cardiaca, su etiología, clasificaciones, tratamiento innovador para tratar la falla cardiaca, entre otros temas que se desarrollan aquí.

\section{Falla cardiaca}

La falla cardiaca es una enfermedad de alta prevalencia a nivel mundial. Se ha convertido en un problema de salud pública en los países industrializados debido a los gastos que genera su atención, principalmente por la alta tasa de rehospitalizaciones y los costos de los tratamientos desarrollados para atender la insuficiencia cardiaca avanzada (5). Lo cual constituye un problema de salud pública de rápido crecimiento debido a sus diversos factores de riesgo que no han sido controlados por las entidades sanitarias.

Por otra parte, la falla cardiaca representa un problema importante de salud pública, ya que afecta cerca de 64.3 millones de pacientes en todo el mundo (6). Sin embargo, en países industrializados como Estados Unidos la estadística muestra que entre el 2,4 y el 2,6\% de la población padecen esta enfermedad; a diferencia de países en vía de desarrollo como en Latinoamérica que las cifras revelan entre el 1,2 y $2,1 \%$ de la población presenta falla cardiaca (7).

En Colombia, según datos estadísticos del ministerio de salud y protección social se estimó que en el año 2012 había 1.100 .000 habitantes que padecían de falla cardiaca, dentro de los cuales el $59.7 \%$ de ese grupo lo conformaron los hombre y el $40.3 \%$ lo conformaron mujeres (8). Entre los años 2009 y 2012 el número de pacientes con falla cardiaca en las salas de urgencia ascendió en un 50\%(9).

La etiología de la falla cardiaca es variada, pero según la bibliografía científica existen tres patologías con las que se encuentra más asociada: la hipertensión arterial, cardiopatía isquémica y miocardiopatía dilatada. También podemos describir que existen diversos factores de riesgo, junto a otras patologías que alteran el normal funcionamiento del corazón incrementando la predisposición a desarrollar falla cardiaca (10).

Éstas causas, sean genéticas, funcionales, infecciosas o estructurales, dividen a la falla cardiaca en dos grupos: las que provocan un impedimento en el vaciado del ventrículo modificando su geometría y de esta manera la fracción de eyección se reduce produciendo insuficiencia sistólica; o la otra forma que se manifiesta sin alterar la fracción de eyección dejándola conservada, a esta se le conoce como insuficiencia diastólica (11).

Por otra parte, estudios recientes han profundizado sobre la fisiopatología de esta enfermedad; se ha 
descubierto que el Péptido Natriurético Auricular tiene propiedades en inhibir el eje renina angiotensina aldosterona, disminuyendo la conducción simpática, con acción diurética y teniendo efectos antihipertróficos (12). Igualmente se ha estudiado que en la falla cardiaca juega un papel importante la Neprilisina; esta es una molécula capaz de inhibir directamente el Péptido Natriurético Auricular (13).

La neprilisina es una metalopeptidasa integral de membrana perteneciente a la familia de peptidasas M13. La neprilisina se encuentra en las membranas de las células nerviosas, renales y células inmunes (14).

A nivel cardiovascular se expresan a nivel del endotelio, regulando la inactivación del péptido natriauricular. Con el bloqueo o inactivación de esta metalopeptidasa de membrana se ha demostrado que induce a natriuresis y vasodilatación. Produciendo efectos antiproliferativos y antimigratorios en el musculo liso a nivel vascular (15).

\section{Valsartán Sacubitril}

A través de múltiples ensayos clínicos realizados acerca del tratamiento de la falla cardiaca, podemos deducir que todavía no existe en el mercado un fármaco que cumpla a cabalidad todas las expectativas para el tratamiento de la falla cardiaca. Aunque cabe resaltar que los medicamentos existentes hasta ahora han mejorado la sobrevida y calidad en muchos pacientes (16) (17).

En diferentes referencias bibliográficas se menciona el uso de los IECAs como fármacos de primera línea en el tratamiento de la falla cardiaca; pero estos medicamentos están relacionados con efectos secundarios como tos, muchas veces asociada a picazón o sensación desagradable en la garganta y angioedema, debido la inhibición de las bradicininas (18).

Recientemente se ha desarrollado un novedoso fármaco llamado LCZ696 que está constituido por una combinación de un ARA II (valsartán) y un inhibidor de neprilisina (Sacubitril). Siendo el valsartán un medicamento clasificado dentro del grupo de los antagonistas de los receptores de angiotensina II (ARA II, en inglés angiotensin II type I receptors blockers o ARBs) entrando en el grupo de fármacos que se especializan en la inhibición del sistema renina angiotensina aldosterona, que en la actualidad se usan para tratar la insuficiencia ventricular con dominancia izquierda, y el inhibidor de neprilisina que actúa contrarrestando todas las acciones de las metaloendopeptidasa como también son conocidas las Neprilisinas (19).

El Valsartán es el fármaco más indicado como opción terapéutica asociado a otros fármacos, debido a que presenta con menos frecuencia angioedema (19). Al Valsartán se le atribuye no causar edema debido a que este fármaco no actúa en la conversión de angiotensina I en angiotensina II, sino que actúa bloqueando la unión de ésta, con los receptores de angiotensina II tipo 1 (AT1) presente en el tejido muscular liso, miocardio y glándula adrenal; inhibiendo así su efecto vasopresor e impidiendo la liberación de aldosterona (20).

Además los ARA II no bloquean la degradación de bradiquinina ni aumenta las acciones de la angiotensina II sobre la presión arterial, que actúa específicamente a través del sistema renal. La angiotensina II interactúa con receptores específicos AT1 de la membrana citoplasmática, provocando el aumento de los niveles citoplasmáticos de $\mathrm{Ca} 2+$, por vía del inositol trifosfato (IP3), y la intensificación de la actividad del intercambiador $\mathrm{Na}+-\mathrm{H}+$ por vía del diacilglicerol (DAG). Mecanismos, causantes de la mayor contractilidad del músculo vascular y de la estimulación de la síntesis de proteína. Adicionalmente la angiotensina II induce la reabsorción de sodio en el túbulo contorneado proximal en el riñón, aumentando el líquido intravascular (21). Por lo cual la inhibición del receptor AT1 por medio del Valsartán permite la disminución del calcio intracelular dando lugar a la relajación del musculo liso vascular y un aumento en la excreción de sodio urinario, por ende disminución del volumen intravascular (22). 
Por su parte, las Neprilisinas son glicoproteínas que abundan en el riñón y que se distribuyen en el borde de cepillo de las células de los túbulos proximales y en el epitelio de los glomérulos (23); esta es una endopeptidasa neutral capaz de separar el lado amino de los residuos hidrofóbicos del propéptido natriurético auricular inhibiendo la transformación a Péptido Auricular Natriurético (24). También es capaz de inactivar muchas hormonas peptídicas como el glucagón, las encefalinas, sustancia $\mathrm{P}$, neurotensina, disminuye la liberación de aldosterona, disminuye el crecimiento celular, inhibe funciones del sistema nervioso simpático (25), disminuye la producción de oxitocina y bradicinina, también está relacionado con la eliminación de los péptidos B-amiloides a nivel encefálico, ayudando a prevenir encefalopatías como el Alzheimer y el Parkinson (26). También se ha demostrado que las Neprilisinas actúan en contra de células invasivas de cáncer de próstata in vitro (27).

Dentro de los péptidos más importantes implicados en la falla cardiaca y que son inactivados por las neprilisinas está el Péptido Auricular Natriurético que se caracteriza por presentar unos potentes efectos cardiovasculares y renales los cuales permiten una opción terapéutica efectiva para el tratamiento de la falla cardiaca (28). El aumento de la concentración del péptido natriurético mediante la inhibición de la neprilisina confiere protección vascular, renal y cardiaca. Cabe resaltar que la inhibición de la neprilisina por sí sola no tiene efecto antihipertensivo significativo; para lograr ver el efecto de acción se tiene que trabajar junto con una inhibición concomitante del sistema renina angiotensina aldosterona (29).

Es conocido que el péptido natriurético auricular estimula la diuresis, la natriuresis, y la vasodilatación con buenos efectos antifibróticos y simpaticolíticos (12). De esta manera, la inhibición de la neprilisina en compañía de la inhibición del sistema renina-angiotensina contribuye a que se regulan los líquidos y electrolitos, por acción directa del péptido natriurético; haciendo que el riñón aumente su tasa de eliminación de sodio y agua, conjuntamente con la disminución de la presión sanguínea que reduce la contracción del músculo liso de los vasos sanguíneos y disminuye la formación de colágeno en las arterias. (28) (30).

Teniendo en cuenta lo anterior se ha diseñado un fármaco conocido como LCZ696 es un inhibidor de neprilisinas de primera clase (Sacubitril) y un inhibidor de receptor de angiotensina (Valsartán) (31). La unión de ambas moléculas farmacológicas se realizó con el fin de minimizar el riesgo de angioedema en los pacientes con falla cardiaca. Según indica el estudio de PARADIGM-HF el inhibidor de neprilisina junto con el valsartán, componen un medicamento innovador con excelentes efectos hemodinámicos y neurohormonales, los cuales favorecen la regulación cardiaca a partir de la inhibición de distintas moléculas (31) (32).

Esta es la nueva estrategia farmacológica coadyuvante en el tratamiento base de la falla cardiaca debido a la acción de estos dos componentes frente a las enzimas que participan en la regulación cardiovascular: neprilisina y enzima convertidora de angiotensina (31). Este mecanismo se ha ensayado con distintos inhibidores de vasopeptidasa como el omapatrilat, el cual ha demostrado una gran reducción de la presión arterial y acción protectora vascular en comparación con otros medicamentos tipo ECA y los bloqueadores de los canales de calcio (33). Sin embargo, este medicamento ha sido asociado a angioedema, debido a la inhibición de tres enzimas que participan en la degradación de las bradiquininas (27).

Dentro del estudio del LCZ696 en el ensayo PARADIGM-HF se obtuvo un resultado con respecto a la reducción porcentual de mortalidad por cualquier factor cardiaco en pacientes con falla cardiaca con fracción de eyección reducida; este fármaco es capaz de reducir considerablemente los niveles de propéptido natriurético y el progreso de la clínica del paciente per se (31).

Con respecto al angioedema, el tratamiento con Sacubitril/Valsartán en el estudio PARADIGM-HF tuvo un efecto negativo en tan solo 10 pacientes de una población total del 4187 pacientes con falla 
cardiaca con fracción de eyección reducida, pero teniendo en cuenta los efectos positivos, estos se vieron manifestados en la poca remodelación de la musculatura cardiaca después de un infarto al miocardio, como también en los resultados que arrojó el estudio PARAMETER que indicaron una mejoría en la disminución de la rigidez de la aorta como tal (31).

El medicamento Sacubitril/Valsartán tiene una vida media en el plasma de aproximadamente 18 horas y es adecuado para administrarlo una vez al día, aunque también puede ser administrado dos veces al día para indicaciones como la falla cardiaca (34); se debe tener precaución a la hora de administrarlo en pacientes que necesiten del tratamiento puesto que se ha estudiado la posibilidad de desarrollar encefalopatías como el Alzheimer y disminución de las funciones cognitivas, puesto que las Neprilisinas se encargan de degradar el péptido beta amiloide que se produce en el cerebro, y al inhibir las Neprilisinas de manera crónica, no será posible la eliminación sistemática de péptido beta amiloide $y$ consecuentemente la acumulación de este péptido en el encéfalo tendrá repercusiones neurológicas. También se ha descubierto que las Neprilisinas ayudan a prevenir la proliferación de células cancerígenas prostáticas in vitro y de igual manera con el cáncer de mama y la incidencia de desarrollar tumores, esto gracias a la inactivación de péptidos como la bradicinina y la endotelina 1 (27).

En ensayos clínicos se ha demostrado que Sacubitril/Valsartán reduce en un 20\% las estancias de los pacientes con falla cardiaca en los hospitales, reduciendo costos de mantenimiento y cuidados clínicos, ya que pacientes con esta dolencia mayores de 65 años de edad, 1 de cada 4 se readmiten con 30 días de incapacidad y al menos 2 de cada 8 necesita seis meses de hospitalización; de esta manera, los pacientes tratados con LCZ696 tuvieron menos requerimiento de ser hospitalizados por una descompensación por falla cardiaca y también necesitaron con menor frecuencia ser readmitidos dentro de los 30 días de cargo hospitalario (31) (35). Estudios recientes muestran una mejora significativa en la funcionalidad ventricular en pacientes con falla cardiaca con fracción de eyección reducida atendidos por fuera del ambiente hospitalario al ser tratados con Sacubitril/Valsartán, esto fue evidenciado imagenológicamente por ecocardiograma (36).

Aunque los IECA y ARA II tienen resultados favorables en la corrección de la fracción de eyección, se ha demostrado que es mucho mejor intervenir en la poscarga temprana que tiene un efecto positivo mayor en el beneficio de la inhibición de Neprilisinas sobre el sistema RAA, pero solo en los grupos de readmisión por falla cardiaca tratados con Sacubitril/Valsartán (35).

Tan alentadores han sido los resultados de los ensayos clínicos fase 3 y 4 del tratamiento con Sacubitril/Valsartán sobre el sistema RAA, que en el año 2017 la American Heart Asociation (AHA) cambio las guías de tratamiento y recomienda utilizar preferiblemente como primera alternativa este tipo de tratamiento en pacientes con falla cardiaca con fracción de eyección reducida (Figura 1) (37).

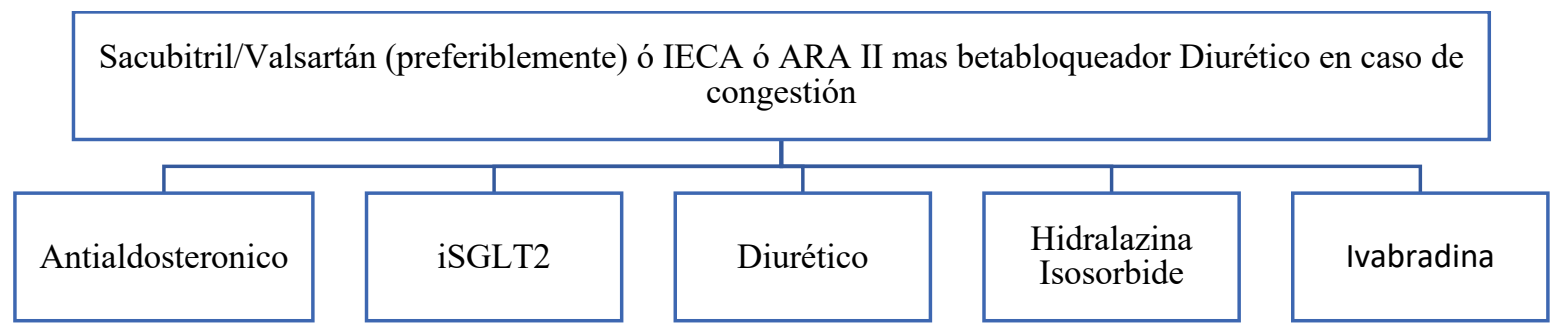

Figura 1. Esquema de tratamiento recomendado. Actualización 2021 del consenso de expertos ACC 2017. Adaptado de: Writing Committee, Maddox TM, Januzzi JL Jr, Allen LA, Breathett K, et al. 2021 Update to the 2017 ACC Expert Consensus Decision Pathway for Optimization of Heart Failure Treatment: Answers to 10 Pivotal Issues About Heart Failure With Reduced Ejection Fraction: A Report of the American College of Cardiology. DOI: 10.1016/j.jacc.2020.11.022 (37) 


\section{CONCLUSIONES}

Sacubitril/Valsartán es un medicamento capaz de disminuir las tasas de hospitalización y la tasa de muerte en pacientes con diagnóstico de falla cardiaca. Su beneficio se ha comprobado en estudios como: PARADIGM-HF (30), TRANSITION (38) Y TITRATION (39) que demuestran la eficacia del medicamento comparado con Enalapril como tratamiento estándar en el manejo de la falla cardiaca, mejorando la calidad de vida de los pacientes y disminuyendo las tasas de rehospitalización.

\section{CONTRIBUCIONES DE LOS AUTORES:} DBY: concepción y diseño del estudio, revisión crítica y aprobación de versión final, responsable de la veracidad e integridad del artículo. DAC y RBM: recolección análisis e interpretación de datos, redacción del borrador del artículo.

\section{CONFLICTOS DE INTERÉS: ninguno que} declarar.

\section{REFERENCIAS}

1. Ulate-Montero G, Ulate-Campos A, Actualización en los mecanismos fisiopatológicos de la insuficiencia cardiaca, AMC, Vol 50 (1), enero-marzo 2008.

2. De La Serna F, Insuficiencia Cardíaca Crónica, Editorial Federación Argentina de Cardiología, 3ra. Edición 2010, Capitulo 4, 2010

3. De La Serna F, Insuficiencia Cardíaca Crónica, Editorial Federación Argentina de Cardiología, 3ra. Edición 2010, capitulo 3, actualizado en 2015.

4. Jagdeep SS Singh, Chim C Lang, Angiotensin receptor-neprilysin Inhibitors Angiotensin receptorneprilysin inhibitors, Vascular Health and Risk Management 2015: 11 283-295.

5. Dickstein K, Cohen-Solal A, Filippatos A. Guía de práctica clínica de la Sociedad Europea de Cardiología (ESC) para el diagnóstico y tratamiento de la insuficiencia cardiaca aguda y crónica (2008), Rev Esp Cardiol. 2008; 61(12): 1329.e1-1329.e70.
6. GBD 2017 Disease and Injury Incidence and Prevalence Collaborators. Global, regional, and national incidence, prevalence, and years lived with disability for 354 diseases and injuries for 195 countries and territories, 1990-2017: a systematic analysis for the Global Burden of Disease Study 2017. Lancet 2018; 392: 1789-1858.

7. Groenewegen A, Rutten FH, Mosterd A, Hoes AW. Epidemiology of heart failure. Eur J Heart Fail. 2020; 22(8): 1342-1356.

8. Jaramillo $\mathrm{C}$, et al., Consenso Colombiano para el diagnóstico y tratamiento de la insuficiencia cardiaca crónica, sociedad colombiana de cardiología y cirugía cardiovascular, 1 edicion, inversiones tecnograficas S.A.S., Bogotá, noviembre de 2014.

9. Tousignant M, Mbuila Mampuya W, Telerehabilitation for patients with heart failure, Cardiovasc Diagn Ther 2015; 5(1): 74-78.

10. Piotr Ponikowski, Adriaan A. Voors, Stefan D. Anker et al, 2016 ESC Guidelines for the diagnosis and treatment of acute and chronic heart failure, European Heart Journal June 8, 2016.

11. Song W, Wang H, Wu Q. Atrial Natriuretic Peptide in Cardiovascular Biology and Disease (NPPA). Gene. 2015; 569(1): 1-6.

12. Jagdeep SS Singh, Chim C Lang, Angiotensin receptor-neprilysin Inhibitors Angiotensin receptorneprilysin inhibitors, Vascular Health and Risk Management 2015: 11 283-295.

13. Barrett AJ, Rawlings ND, O'Brien EA. The MEROPS database as a protease information system. J Struct Biol 2001;1 34: 95-102.

14. Nalivaeva, N. N., Zhuravin, I. A., \& Turner, A. J. (2020). Neprilysin expression and functions in development, ageing and disease. Mechanisms of ageing and development, 192, 111363. https://doi.org/10.1016/j.mad.2020.111363

15. Salazar, J., Rojas-Quintero, J., Cano, C., Pérez, J. L., Ramírez, P., Carrasquero, R., Torres, W., Espinoza, C., Chacín-González, M., \& Bermúdez, V. (2020). Neprilysin: A Potential Therapeutic Target of Arterial Hypertension?. Current cardiology reviews, 16(1), 25- 
35.

https://doi.org/10.2174/1573403X15666190625160352

16. Chaves G, Diaztagle J, Vargas V, Mejía M, Sprockel $\mathrm{J}$, Hernández J, Cumplimiento de guías en pacientes hospitalizados con falla cardiaca ¿Cómo estamos? Acta Médica Colombiana 2014, 39 (Enero-Marzo).

17. Von Lueder TG, Atar D, Krum H. Current role of neprilysin inhibitors in hypertension and heart failure. Pharmacol Ther 144(1): 41-49 (2014).

18. García S, Parodi R, Tos y angioedema en pacientes tratados con inhibidores de la enzima convertidora de la angiotensina siempre es culpable la medicación, Rev. argent. cardiol. vol.79 no.2 Ciudad Autónoma de Buenos Aires mar./abr. 2011.

19. Braunwald $\mathrm{E}$. The path to an angiotensin receptor antagonist-neprilysin inhibitor in the treatment of heart failure. J Am Coll Cardiol. 2015; 65: 1029-1041.

20. Grothusen A, Divchev D, Luchtefeld M, Schieffer B. Angiotensin II type 1 receptor blockade: high hopes sent back to reality? Minerva Cardioangiol. 2009 Dec; 57(6): 773-85.

21. Forrester, S. J., Booz, G. W., Sigmund, C. D., Coffman, T. M., Kawai, T., Rizzo, V., Scalia, R., \& Eguchi, S. (2018). Angiotensin II Signal Transduction: An Update on Mechanisms of Physiology and Pathophysiology. Physiological reviews, 98(3), 16271738. https://doi.org/10.1152/physrev.00038.2017

22. Kinoshita H, Kuwahara K, Nishida M, Jian Z, Rong X, Kiyonaka S, Kuwabara Y, Kurose H, Inoue R, Mori Y, Li Y, Nakagawa Y, Usami S, Fujiwara M, Yamada Y, Minami T, Ueshima K, Nakao K. Inhibition of TRPC6 channel activity contributes to the antihypertrophic effects of natriuretic peptides-guanylyl cyclase-A signaling in the heart. Circ Res. 2010 Jun 25; 106(12): 1849-60.

23. Gaggin H.K., Januzzi J.L. Jr. (2013) Biomarkers and diagnostics in heart failure. Biochim Biophys Acta 1832: 2442-2450.

24. Giuseppe Lippi, Fabian Sanchis-Gomar, Monitoring B-type natriuretic peptide in patients undergoing therapy with neprilysin inhibitors. An emerging challenge, International Journal of Cardiology 219 (2016) 111-114.
25. Pacheco-Quinto J, Herdt A, Eckman CB, Eckman EA. Endothelin-converting enzymes and related metalloproteases in Alzheimer's disease. J Alzheimers Dis. 2013; 33 Suppl 1: S101-10.

26. Galli A, Lombardi F, Neprilysin Inhibition for Heart Failure, The new england journal of medicine, nengljmed 371;24 nejm.org december 11, 2014.

27. McMurray JJ, Packer M, Desai AS, Gong J, Lefkowitz MP, Rizkala AR, Rouleau JL, Shi VC, Solomon SD, Swedberg K, Zile MR, PARADIGM-HF Investigators and Committees. Angiotensin-neprilysin inhibition versus enalapril in heart failure. N Engl J Med. 2014 Sep 11; 371(11): 993-1004.

28. to S, Satoh M, Tamaki Y., Safety and efficacy of LCZ696, a first-in-class angiotensin receptor neprilysin inhibitor, in Japanese patients with hypertension and renal dysfunction, The Japanese Society of Hypertension, Hypertension Research (2015) 38, 269-275.

29. Adriaan A. Voors et al, Renal effects of neprilysin inhibitor LCZ696 in patients with HFpEF, European Journal of Heart Failure (C) 2015 European Society of Cardiology, European Journal of Heart Failure (2015) 17, 510-517.

30. McMurray JJ, Packer M, Desai AS, Gong J, Lefkowitz MP, Rizkala AR, Rouleau J, Shi VC, Solomon SD, Swedberg K, Zile MR; Dual angiotensin receptor and neprilysin inhibition as an alternative to angiotensinconverting enzyme inhibition in patients with chronic systolic heart failure: rationale for and design of the Prospective comparison of ARNI with ACEI to Determine Impact on Global Mortality and morbidity in Heart Failure trial (PARADIGM-HF). Eur J Heart Fail. 2013; 15: 1062-1073.

31. Sadayoshi Ito et al, Safety and efficacy of LCZ696, a first-in-class angiotensin receptor neprilysin inhibitor, in Japanese patients with hypertension and renal dysfunction, Hypertension Research (2015) 38, 269-275.

32. Dalzell JR, Seed A, Berry C, Whelan CJ, Petrie MC, Padmanabhan N, Clarke A, Biggerstaff F, Hillier C, McMurray JJ. Effects of neutral endopeptidase (neprilysin) inhibition on the response to other vasoactive peptides in small human resistance arteries: studies with thiorphan and omapatrilat. Cardiovasc Ther. 2014 Feb; 32(1): 13-8. 
33. Jagdeep SS Singh, Chim C Lang, Angiotensin receptor-neprilysin Inhibitors Angiotensin receptorneprilysin inhibitors, Vascular Health and Risk Management 2015:11 283-295.

34. Akshay S. Desai et al, Influence of Sacubitril Valsartan (LCZ696) on 30-Day Readmission After Heart Failure Hospitalization, JACC VOL. 68, NO.3, 2016, JULY 19, 2016: 241-8.

35. Dargad RR, Prajapati MR, Dargad RR, Parekh JD. Sacubitril/valsartan: A novel angiotensin receptorneprilysin inhibitor. Indian Heart J. 2018; 70 Suppl 1(Suppl 1): S102-S110.

36. Ganesananthan S, Shah N, Shah P, et al. Real-world treatment switching to sacubitril/valsartan in patients with heart failure with reduced ejection fraction: A cohort study. Open Heart. 2020; 7(2): e001305.
37. Writing Committee, Maddox TM, Januzzi JL Jr, Allen LA, Breathett K, et al. 2021 Update to the 2017 ACC Expert Consensus Decision Pathway for Optimization of Heart Failure Treatment: Answers to 10 Pivotal Issues About Heart Failure With Reduced Ejection Fraction: A Report of the American College of Cardiology Solution Set Oversight Committee. J Am Coll Cardiol. 2021 Jan 4: S0735-1097 (20) 37867-0.

38. Wachter R, Senni M, Belohlavek J, et al. TRANSITION Investigators. Initiation of sacubitril/valsartan in haemodynamically stabilised heart failure patients in hospital or early after discharge: primary results of the randomised TRANSITION study. Eur J Heart Fail. 2019 Aug; 21(8): 998-1007.

39. Senni M, McMurray JJ, Wachter R, et al. Initiating sacubitril/valsartan (LCZ696) in heart failure: results of TITRATION, a double-blind, randomized comparison of two uptitration regimens. Eur J Heart Fail. 2016 Sep; 18(9): 1193-202. 